\title{
Local Practices, Problems and Thoughts from Governance Perspective on Affiliation of Regional Prestigious Schools
}

\author{
Da Wang \\ Education Research Institute of Gongshu District, Hangzhou, China \\ Email:gsjywd@163.com
}

How to cite this paper: Wang, D. (2020). Local Practices, Problems and Thoughts from Governance Perspective on Affiliation of Regional Prestigious Schools. Open Journal of Leadership, 9, 1-10. https://doi.org/10.4236/oj1.2020.91001

Received: December 29, 2019

Accepted: January 19, 2020

Published: January 22, 2020

Copyright $\odot 2020$ by author(s) and Scientific Research Publishing Inc. This work is licensed under the Creative Commons Attribution International License (CC BY 4.0).

http://creativecommons.org/licenses/by/4.0/

\section{(c) (i) Open Access}

\begin{abstract}
The aim of this research was to provide a new development direction and guidance for the construction of prestigious school group by analyzing the advantages and disadvantages of the development of Hangzhou prestigious school group in the past 20 years. The affiliation construction of prestigious schools is one of the significant approaches to promote good-quality and well-balanced growth of regional basic education. This paper, using the concept of "governance", analyzes the condition of promoting affiliation construction of prestigious schools in Gongshu District, summarizes the development models for high-quality regional education such as "prestigious school+" version 1.0, "school development union" version 2.0 and "new prestigious school group" version 3.0. It also examines problems in practices and presents thoughts over future development.
\end{abstract}

\section{Keywords}

Affiliation of Prestigious Schools, Education Governance, Good-Quality and Well-Balanced

In 1999, Hangzhou became a forerunner in China to launch affiliation of public schools, which have been underway for 20 years by now. What are innovative measures of operation mechanism during the past 20 years? What are the goals of regional education development? How can we follow these goals and give full play to the resource superiority of affiliation of prestigious schools? This paper tried to review and examine the affiliation construction of prestigious schools from governance perspective. We hope that this can help us to know the problems deeply and obtain certain references and experience accordingly. 


\section{Introduction of "Governance" Concept into Affiliation of Regional Prestigious Schools}

As defined in the report of "Our Global Neighborhood" released by the Commission on Global Governance in 1995, the term "governance" means: the aggregation of various means to manage common issues by public or private individuals and organizations as well as the continuous process of coordinating conflicting or different stakeholders into joint actions (The Comission on Global Governance, 1995). This definition of governance not only focuses on its publicness, humanity and harmony but also emphasizes on the establishment and improvement of public management systems featuring co-governance and co-sharing.

As applied in education, education governance refers to the establishment of systematic, complete, scientific, standardized and effective mechanisms through continuous interaction such as dialogue, negotiation and discussion by stakeholders such as government organizations, schools, social organizations and citizens, which aims to realize proper division of functions among governing subjects, promote separation among "supervision, operation and evaluation". Meanwhile, it aims to construct a new relationship among government, school and society by certain mechanism arrangement to jointly manage public issues of education and realize "coordinated governance" by multiple subjects (Zhao \& Dai, 2017). With actual conditions taken into consideration, the concept of "governance" in constructing affiliation of regional prestigious schools should have the following features:

First, the participation of multiple subjects should be emphasized. The subjects of education governance include organizations such as governments, schools and communities as well as individuals such as principals, teachers, students and parents. From the perspective of "governance" during the construction of affiliation of regional prestigious schools, there is a higher demand for participation of multiple subjects, as well as a closer relationship among stakeholders in interaction, cooperation, sharing and symbiosis. Thanks to factors such as resources guarantee by government, operation by school management and joint participation by social circles, we may boost the good-quality and well-balanced growth of regional education resources while fostering positive and mutually-beneficial interaction among teachers, students, parents and schools.

Second, interests of relevant stakeholders should be coordinated. From governance perspective, as various subjects are engaged in affiliation of regional prestigious schools, it is necessary to "coordinate" the pursuit of their own interests. It must be stressed that all stakeholders, as they consider their own interests, must give consideration and acknowledgement to interests of other parties (Wang, 2007). It is necessary to share resources of prestigious schools in all aspects and by all people during such construction.

Third, transparency under the sunshine should be highlighted. The modern school governance calls for three conversions: from rule by people to rule of law, from centralization of power to separation of power, from self-governance 
to co-governance (Yang, 2019). For market-based allocation of good resources during affiliation of regional prestigious schools, it is vital to establish a transparent mechanism under the sunshine for co-construction, co-governance and co-sharing among different campuses within groups as well as between the headquarters and member schools.

Fourth, progress in a fair and just way should be guaranteed. As education governance enters a new era, the school development in the new era is featured as law-based, standardized and professional. To safeguard the fair and just progress of such construction, important measures should be adopted, such as the principle to separate "management, operation and evaluation" as well as the introduction of the third-party in evaluation. Such fair, just and objective evaluation is indispensable in assessing growth of new campuses and their potential of independent development away from the parent schools.

Fifth, the intention of autonomous school operation should be fulfilled. Prestigious school groups should be governed by constitution. Various campuses or member schools within a prestigious school group enjoy autonomy in operation and necessary control over human resources, financial resources and materials, which serves as important measures to motivate each education subject in the affiliation of prestigious schools. The option for each education subject within the group should be respected for differentiated and characteristic development, avoiding repetitive sameness after forming groups of prestigious schools.

Generally speaking, the concept of "governance" requires the participation of multiple subjects and the coordination of interests of multiple parties in the construction of regional prestigious universities. At the same time, it is necessary to respect the wishes of each school during the construction process and to make the construction process plan open, transparent and fair.

\section{Approaches in Implementing Affiliation of Regional Prestigious Schools}

Since such a practice was started in Gongshu District, three versions of affiliation of prestigious schools emerged one after another, leading to the current "new prestigious school group version 3.0". Now, let's briefly review the relevant practices of these versions. Based on this, we will compare the similarities and differences to help us to further understand it.

\subsection{Version 1.0: Incubating Four Models of "Prestigious Schools+"}

At the beginning of such construction, in order to boost the well-balanced development of regional good-quality education resources, with the promotion from administrations, one or several prestigious schools take the lead to establish a new contractual form of school organization through two ways: either supporting the weak partner by the strong partner or complementing each other's advantages. During about 10 years since 2004, based on practice, various modes of affiliation are adopted in Gongshu District. 
The first is "prestigious school + new school", as good public schools play a leading role in establishing new schools and forming groups. To promote the overall growth, the group adopts unified management internally and integrated operations, such as restructuring management and resources, exchange of teachers and management, unified evaluation, reward and punishment.

The second is "prestigious school + ordinary school", as good schools lead ordinary schools to form groups. By sharing and restructuring within the group, the overall education quality improves, as close cooperation is established internally, such as interconnection of management, exchange of teachers, interaction of research and training, joint progress of quality. During the process, the unique features of ordinary schools are inherited and boosted to promote differentiated growth among member schools with distinctive features.

The third is "private school + public school", as good private schools take the lead in organizing education groups, managing new public schools and ordinary public schools. Good private schools, mainly by exporting education philosophy, culture, values and experience, carry out multi-level and overall cooperation in management and teaching resources to promote synergized growth of public and private schools.

The fourth is "outsider schools + local schools", as prestigious schools in other parts of China, with their strong brands, lead local schools informing education groups to realize deep cooperation in brand sharing, management interconnection and joint teachers training.

Meanwhile, cooperation is further enhanced with universities, colleges and research institutes to continuously uplift the standard of affiliation of prestigious schools, with emphasis on curriculum reform, teachers training, internationalization and information (Yu \& Ruan, 2017).

\subsection{Version 2.0: School Development League for Enhanced Strength}

When the development of good-quality and well-balanced regional education meets its bottleneck, how can we enable sustained growth based on high level, restructure good resources, make good schools lead weak schools towards stronger capability, promote quick expansion of good resources and satisfy the demand for good education from the general public? Initiated by the district education bureau in 2014, several schools form "school development league" based on principles such as autonomous participation, equality-based grouping, promotion of distinctive features and bundled evaluation.

A new growth opportunity with local features emerges during the construction of affiliation of prestigious schools. The influence of prestigious schools as leaders is fully utilized to boost schools in the league, with the system of joint discussion of plans, joint sharing of resources and joint participation of programs within the league. As shown in Figure 1, the operation of such a development league gives full play to the "governance" mechanism, as the league leader, or the convener of the league, shoulders responsibility, while the district 


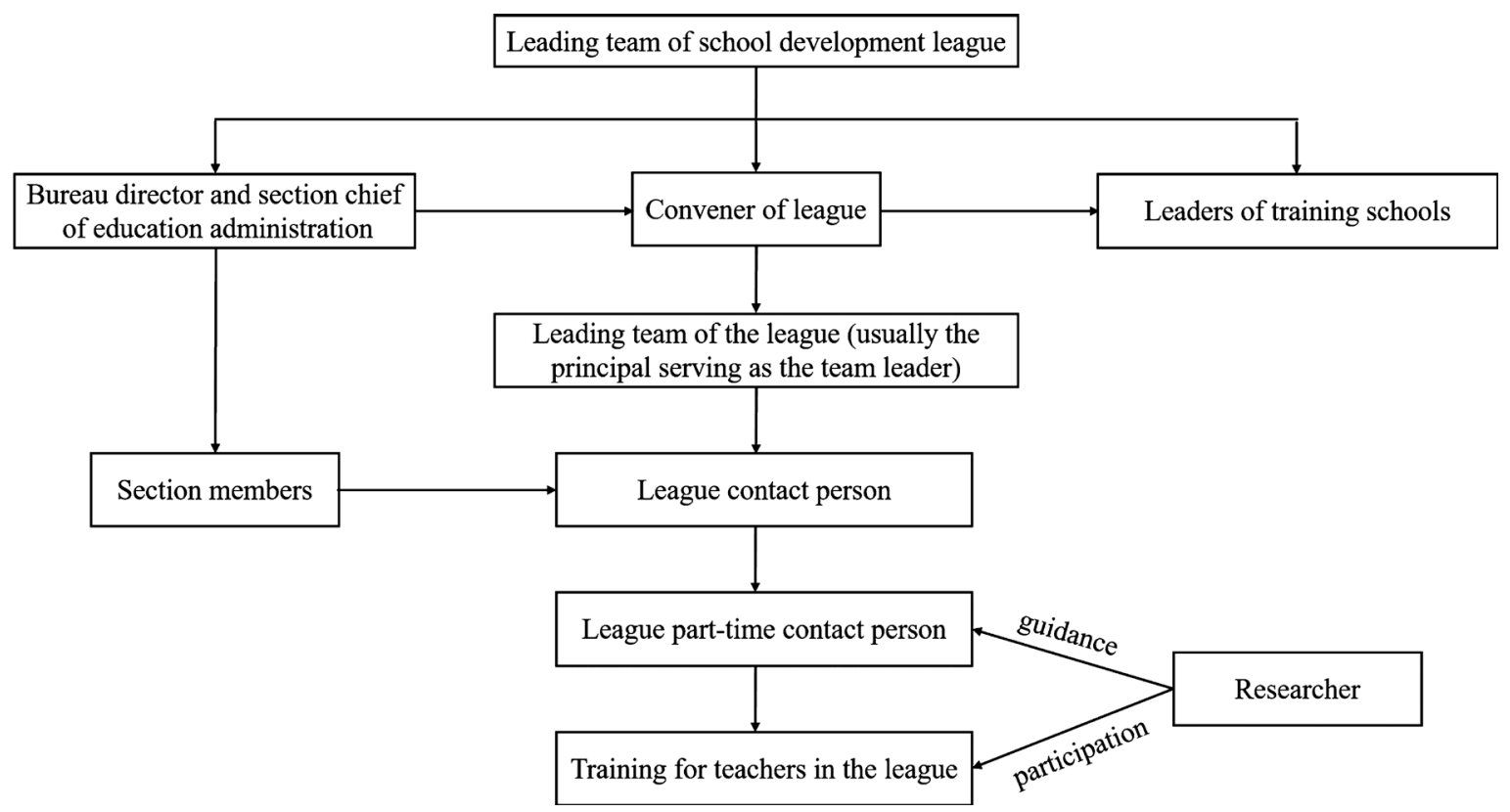

Figure 1. Operation of school development league in Gongshu District.

education bureau and related departments participate only in the capacity of "liaison" to learn progress at certain stages and offer assistance only on the occasions of difficulties and challenges. Within the district, all ten school development leagues have respective leading teams composed of principals from each member schools. As all significant issues must be subject to leading teams for discussion before implementation, the leagues achieve co-governance and co-sharing (Xie, 2015). To improve capability of teachers in school development leagues, the district-level teaching and research are shifted downward to league-level. Each league seeks excellent teachers to serve as part-time researchers, whereas district-level researchers serve as leaders of league-level research activities, thus achieving cooperative development for teachers in the leagues.

\subsection{Version 3.0: Highlighting Brands by "Double-Prestigious Project"}

In the new era, Gongshu District, already a district of generally good balance in basic education, is advancing towards higher level of good-quality and well-balanced regional education, with the new task of reaching the education target of "fairness and quality". In 2018, the version 3.0 of "new prestigious school group" with a stronger sense of gain for the new era was launched together with promotion of "double-prestigious project", aiming to further deepen affiliation strategy of prestigious schools, encourage the influence of good-quality education resources, enhance brand construction of prestigious schools and enable the general public to enjoy quality education resources nearby. The first is "prestigious schools leading project". Guided by the "10 prestigious school groups of basic education", a result of the previous work, good education resources are vigorously encouraged to cover the new areas in the north of Gongshu District. 
Among the new schools in the north, "new round of affiliation of prestigious schools" are underway, which promotes the construction and development of those schools in the north at high starting point and high level, by means of exporting advanced education philosophy, successful management mode, effective classroom teaching and excellent teachers. As an iconic action in such a project, "Highlighting Brand" was launched by a new school named Yucai Dengyun Primary School, a member of Yucai Middle School Education Group. Soon afterwards, all "10 prestigious school groups of basic education" in the district as well as their member schools all highlighted their brands and concepts.

The general public, directly witnessing the impact of the progress during the past 20 years, may deeply feel the increasing number of good schools nearby. The second is "prestigious schools fostering project", which focuses on improving ordinary schools around the general public living in the south part of Gongshu District. While stressing the creation of good-quality schools and under the school constitution of multiple legal persons, schools are encouraged to highlight distinctive education features, improve education quality and "governance" mechanism in order to maximize excellent education resources in the district. Recently Desheng Primary School took over the vacated school buildings in the old residential area of South Daguan to enhance its brand impact, which served as a starting point to further promote the "prestigious schools fostering project" around the general public.

Moreover, during such construction process, we emphasize advanced school infrastructure and facilities, promote the integration of local experts and external experts, guide high-quality school development as devised by Gongshu model according to first-rate standards in China, such as "Construction Standard of Schools (Kindergartens)", "Rules on Colors in Construction of Schools (Kindergartens)". Meanwhile, in order to improve teaching resources of new prestigious school groups, cooperation with high-level training agencies in China is underway to hold high-level classes with the theme of "future principals" and "future teachers".

In order to better understand the differences between the different versions of the practice path for the development of regional prestigious schools, we made a simple comparison. I hope to further clarify the role of different versions in the development of regional prestigious schools through the comparison between the versions.

First, the starting point of these three versions is to promote the development of regional prestigious schools. However, there are differences in the number of schools between versions 1, 2 and 3. In the first version, many schools participated together, and the number was huge. Further development of the group development of multiple schools in version 2 highlights the role of group units. Finally, the development of version 3 further selects outstanding schools in the group unit as the leader to play a leading role.

Second, these three editions have played a significant role in promoting the 
development of the school. However, there are differences in the roles of the three versions. In version 1, it mainly promotes the development of education and teaching and teachers in schools in the region. In version 2, it is mainly based on the reconstruction of high-quality resources based on 1 , so that the superior schools bring weak schools, and the method of turning weak schools into strong schools further promotes the rapid expansion of high-quality resources. In version 3, the main purpose is to further promote regional schools to a higher level of high-quality regional education and achieve a balanced and quality education goal.

\section{Solutions on Problems Concerning Affiliation of Regional Prestigious Schools from Governance Perspective}

Analyzing cases of regional education development in Gongshu District from governance perspective, we may find problems still exist in spite of affiliation of prestigious schools that originally aimed at good-quality and well-balance growth of regional education.

\subsection{We Need to Enhance the Win-Win Cooperation Philosophy during Affiliation of Prestigious Schools}

The precondition of education governance lies on consensus about specific ideology among decision-makers (Lu et al., 2019). The affiliation of prestigious schools is not only an objective demand for good-quality and well-balanced growth of regional education but also a requirement for standardized operation during school management of multiple campuses and multiple legal persons.

Among the cases of regional education development in Gongshu District, during the practice of "prestigious school leading project", some schools, for the purpose of future independent school operation, use independent legal persons (school-level leaders from the headquarter school) in registration for new campuses as they take the leading role in building new campuses. In actual practice, some legal persons, considering future independent development and focusing on their own agenda, often overplay individuality of new campuses but fail to fully carry forward the philosophy of prestigious school groups.

As a good school tries to boost the development of a nearby ordinary school during "prestigious school fostering project", the two schools may differ sharply in historical and cultural heritage, development path and working style of teachers. In a prestigious school education group with multiple legal persons, each party retains independent financial administration rights. Upholding the same philosophy, two schools in cooperation and development may still face conflicts and even fail to reach consensus after three years. Solutions for these problems are to be explored in future practice.

\subsection{We Need to Strengthen the Universal Coverage of Good Resources during Affiliation of Prestigious Schools}

The implementation of education governance requires publicness of resources, 
whereas the balance of regional education is reflected by the publicness of resource allocation. The concentration of good resources in a handful of schools is criticized, as the general public call for good-quality education nearby in the new era.

Some measures are taken in Gongshu District to realize the universal coverage of prestigious schools resources: all newly-built schools are operated by prestigious schools as leaders, following affiliation pattern under the new situation of urbanization in North Gongshu District. Some good resources already materialize, such as No. 2 Hongying Kindergarten in Taoyuan New Area, new campus of Maiyuqiao Primary School and North Campus of Yucai School in Grand Canal New City.

However, as over 20 new campuses will be built in the north new area in recent 10 years, the good resources are far from enough, even with full dedication of "10 prestigious school groups of basic education". It is a constant task during development to accelerate fostering schools of core brands and enable universal coverage of more and more good resources. During affiliation of prestigious schools, we also face the challenge whether good education resources in the south area will be attenuated the same way as milk is diluted.

\subsection{We Need to Improve the Synergized Governance System for Affiliation of Prestigious Schools}

The "governance" mechanism emphasizes orderly participation by multiple parties, with negotiation as a important measure to handle common issues. So far among principals of the same group during such a process, the co-governance capabilities are still doubtful and the necessary mechanism to solve problems through negotiation is still absent. We face the urgent challenge to form a synergized governance system internally and externally.

In practice, such a process is promoted, to a large extent, by principals of good schools or education administrative authorities. In theory, the group constitution can solve mechanism problems in "governance", but in practice some principals have weak rule-based and contract-based awareness. When reviewing group constitutions in work, the author once found that the chairman of a prestigious school group revised the constitution as "the chairman has one-vote veto power", which was astonishing for his weak law-based consciousness putting the individual power beyond organization. Since the mechanism from governance perspective is so weak even in the main urban district of a sub-provincial city, there is still a long way to go for "governance" to prevail.

\section{Future for Affiliation of Prestigious School from Governance Perspective}

In the third part, this paper mainly discusses the problems and possible strategies to solve the problems of regional famous school grouping from the current governance perspective. In this part, the main problem struck by the third part is the method of the specificity of the future construction and development. By 
analyzing affiliation of regional prestigious schools from the perspective of education governance as well as reflecting on development modes and problems, we may enhance innovation capabilities during such construction and explore future development ideas. Based on the concept of "governance", we may promote such construction in the following aspects such as concepts, strength and mechanism.

\subsection{The First Is to Establish Development Concept and Build Win-Win Cooperation Models in Construction of Affiliation of Prestigious Schools}

We should truly focus on the future growth of schools and personal development of teachers, respect all participants in such process, guarantee their legal right of speech, provide both principals and teachers with growth room and targets, actively enhance sense of gain for participants and form synergy of co-governance and co-sharing during such construction.

\subsection{The Second Is to Enhance Resources Input and Promote Self-Sustained Growth of Prestigious School Groups}

It is critical whether good education resources during such construction may grow anew after reproduction. When mature campuses or member schools within the group are capable of independent growth under proper conditions, regional education administration should enhance resources input in time, establish development platforms, enable new independent schools to grow into good education resources to lead other new campuses, thus realizing universal coverage of good regional education around the general public.

\subsection{The Third Is to Improve "Governance" Mechanism and Establish an Interactive Development System Based on Negotiation}

From "governance" perspective, the less administrative directive, the better. We should, under the guidance of administrative authorities, establish mechanisms on construction plan, constitution review, KPI incentives urge each school groups to formulate and improve their own development plans, further improve group constitution based on rule of law and truly form a system of negotiation, interaction and development inside prestigious school groups.

In conclusion, during the affiliation of regional prestigious schools, we first need to tackle the issue of concepts of people. When people reach consensus, individual strength is transformed into collective action. Guided by culture, values and concepts, such process may help all participants improve themselves. Secondly, we need to tackle the issue of resources input. For the affiliation of prestigious to survive and thrive, continuous input is necessary to nurture new growth points and form growth sources. Lastly, we need to tackle the people-to-people issue. The good-quality and well-balanced regional education can enjoy sustained growth with the help of driving forces for "governance" in affiliation of presti- 
gious schools, such as democratic environment, negotiation mechanism and institutional constraint.

\section{Conclusion}

This research has combed the influence of the concept of "governance" on the construction of prestigious school groups in detail, the problems existing in the construction of prestigious school groups, and the future development direction of prestigious regional school groups. We find that the concept of "governance" helps to promote the construction of prestigious school groups. At present, there are problems such as unclear concepts, unreasonable resource allocation, and insufficient cooperation between schools. In future development, we need to strengthen resource allocation and strengthen cooperation between schools.

\section{Conflicts of Interest}

The author declares no conflicts of interest regarding the publication of this paper.

\section{References}

The Comission on Global Governance (1995). Our Global Neighborhood (the Basic Vision), Geneva.

Lu, L.-T., Wang, H.-Y., \& Gao, F. (2019). Curriculum Construction of Study Tour from Governance Perspective: Models, Problems and Reflections. Management on Primary and Secondary Schools, 7, 53-82.

Wang, X.-H. (2007). A Theoretical Construction of Education Governance. Journal of Beijing Normal University, 4, 5-14.

Xie, Q. (2015). Preliminary Exploration on Balanced Development at High Level of Regional Education during Post Affiliation of Prestigious Schools-Gongshu District in Hangzhou as a Sample. Shanghai Education Research, 9, 57-59.

Yang, X.-M. (2019). Quality Symbiosis: Inquiry on Values and Choice of Approach in Restarting School Affiliation. Management on Primary and Secondary Schools, 5, 347-367.

Yu, X.-D., \& Ruan, Q.-Q. (2017). New Affiliation of Prestigious Schools: "Hangzhou Practice" in Supply Side Reform on Urban Education. People's Education, 11, 21-25.

Zhao, L.-L., \& Dai, R.-H. (2017). Regional Education Governance: Content, Target and Approach. Education Science Research, 9, 25-28. 